\title{
Predicting Numerical Comparison using Neural Networks and Electrophysiological data
}

\author{
Richard W Prather \\ Sara Heverly-Fitt \\ Department of Human Development and Quantitative Methodology \\ University of Maryland, College Park
}

\begin{abstract}
The effectiveness of cognitive interventions is dependent on researchers' ability to predict individual participants' behavior. In this study we present a novel computational model design that uses both behavioral and electrophysiological input to predict participants' behavior the numerical comparison task. We focus on the numerical comparison task as performance on this task is used for the Numeracy construct which predicts important mathematical outcomes. We model participants' behavior using independent model instantiations that are optimized for each participant using an evolutionary algorithm. We demonstrate that the use of electrophysiological data, at the individual trial level, can significantly improve the model's accuracy. We discuss both the potential and limitations of the current paradigm in developing training regimens for children with early math difficulty.
\end{abstract}




\section{Introduction}

Interest in the development of interventions and training paradigms cuts across areas of cognition such as language learning, memory, numerical cognition. The goal of such interventions typically is to improve learners' performance on a target task (Park, Bermudez, Roberts, \& Brannon, 2016; Park \& Brannon, 2014; Prather \& Alibali, 2011), or in the case of older adults prevent decline (e.g., Anguera et al., 2013). The design of effective interventions requires the prediction of learners' behavior. The current study evaluates a predictive computational model of learners' behavior on a numerical task. A significant limitation in intervention design is the individual variation in participant behavior. A particular training sequence or task that is effective for participants of average skill may not be for participants with lownumeracy. There has been an increase in the automatization (e.g., Iuculano et al., 2015) and individualization of interventions (e.g., Cohen Kadosh, Dowker, Heine, Kaufmann, \& Kucian, 2013; Vanlehn, 2011).

As an initial step in creating individualized training procedures, we developed a computational method for single-trial predictions of participants' behavior. We focus on evaluating the canonical nonsymbolic numerical comparison task. Participant's skill in this task is used to index their numeracy; a construct that has been shown to correlate with important outcomes related to math performance (e.g., Libertus, Feigenson, \& Halberda, 2013; Libertus, Odic, \& Halberda, 2012; Mazzocco, Feigenson, \& Halberda, 2011). Participants' skill in non-symbolic numerical comparison is correlated with their skill on symbolic arithmetic (e.g., Chen \& Li, 2014; Fazio, Bailey, Thompson, \& Siegler, 2014). The use of nonsymbolic numerical comparison as a training task that may transfer to symbolic arithmetic has mixed results (Park et al., 2016; Park \& Brannon, 2013, 2014). 


\section{Behavioral Experiment}

In this experiment, adults completed the numerical comparison task while electrophysiological measures were taken. The event related potentials associated with numerical comparison were then used as input in model optimization. The neural correlates of the comparison task, as seen with electroencephalography, include a neural distance effect where the amplitude of event related potentials vary based on the distance of the comparison(e.g., Ben-Shalom, Berger, \& Henik, 2013; Heine, Tamm, Wissmann, \& Jacobs, 2011; Jiang et al., 2010). In parietal areas typically associated with numerical tasks, event related potentials are larger for close comparisons and smaller for far comparisons. This effect is typically reported between 300 and 500 milliseconds, though there is some variation in the literature.

\section{Behavioral Method}

Participants. Participants $(n=35)$ were adults recruited through a university participant pool. Protocols were approved by the University of Maryland Internal Review Board.

Procedure. We evaluated participants' performance on the non-symbolic numerical comparison task while measuring neural correlates via EEG. Participants were instructed that they would complete a computer-based task while simultaneous EEG recordings were taken. After a brief set up to initialize the EEG participants began the behavioral task. Instructions for the task were given orally and again written

on the screen. Participants' responses were via a key press. The experimental session was approximately 45 minutes. 
Task and Stimuli. Stimuli were 90 visually presented pairs of shape arrays with a midline separator. Shape arrays ranged in number from 23 to 111 (see Appendix A) with a maximum ratio difference of 2.6. Participants were instructed to indicate which side contained more shapes via a button press. Stimuli were displayed for 2 seconds after which the screen was blank. There was no response time limit; participants were instructed to respond as quickly as possible.

Electrophysiological Recordings Specifications. The recording was implemented using a 32-channel EEG cap. Twenty nine tin electrodes were held in place on the scalp by an elastic cap 1 (Electro - Cap International, Inc., Eaton, OH) in a 10 - 20 configuration (01, FP2, O2, P7, 2 P3, Pz, P4, P8, TP7, Cp3, CPz, CP4, TP8, T7, C3, Cz, C4, T8, FT7, FC3, FCz, FC4, FT8, 3 F7, F3, Fz, F4, F8, FP1). Based on prior research we limited the analysis to channels in the left parietal area (P3, P7, CP3, TP7). Bipolar electrodes were placed above and below the left eye and 4 at the outer canthus of the right and left eyes to monitor vertical and horizontal eye 5 movements. Additional electrodes were placed over the left and right mastoids. Scalp 6 electrodes were referenced online to the left mastoid and re - referenced offline to the 7 average of left and right mastoids. Impedances were maintained at less than $5 \mathrm{k} \Omega$ for all 8 scalp electrode sites, less than $2 \mathrm{k} \Omega$ for mastoid sites, and less than $10 \mathrm{k} \Omega$ for ocular 9 electrodes. The EEG signal was amplified by a NeuroScan SynAmps® Model 508310 (NeuroScan, Inc., Charlotte, NC) with a bandpass of 0.05 - 100 $\mathrm{Hz}$ and was continuously 11 sampled at $500 \mathrm{~Hz}$ by an analog - to - digital converter.

\section{Results}


Behavioral results. Participant performance on the numerical comparison task ranged from 83\% to 97\% correct with the overall performance at $89 \%$. Median reaction times for participants ranged from $352 \mathrm{~ms}$ to $1604 \mathrm{~ms}$. Collapsing across all participants the correlation between trial ratio difference and percent correct was non-significant, $\mathrm{r}=0.22, \mathrm{t}(88)=2.15, \mathrm{p}=0.03$ likely due to a ceiling effect. Correlation between reaction time and trial ratio difference was $r=-0.295, t(88)=2.89, p=0.004$.

Electrophysiological results. We evaluated if there was an ERP distance effect in parietal areas. Mean ERP amplitude within the time window of 280-380ms was extracted. Trials were binned into equal sized groups of large, medium or small comparison distances. These ratio distances corresponded to greater than 1.9, less than 1.4 or in between 1.9 and 1.4. We compared the mean ERP amplitude for large and small comparison distances. Data were analyzed using repeated measures ANOVA, with trial comparison distance being the within-subjects variable. For this analysis, we only include participants in which at least $90 \%$ of trials produced a useable ERP $(n=26)$. Some trials produced artifacts or extreme outliers. Our analysis shows that when evaluating data from two parietal channels, there is a significant difference in ERP amplitude between large and small comparison differences. We found a significant effect of ratio difference on mean ERP amplitude for the P3 and CP3, $F(1,25)=6.33, p=.018$ and $F(1,25)=4.58, p=$ .042 respectively. This analysis of the neural distance effect is consistent with prior results in terms of EEG channel location, ERP time window, and trial comparison.

The results replicate the neural distance effect (e.g., Ben-Shalom, Berger, \& Henik, 2013; Heine, Tamm, Wissmann, \& Jacobs, 2011; Jiang et al., 2010). We find that event related potentials for two parietal channels (P3 and CP3) significantly varied for close numerical comparisons versus far comparisons. For the remaining channels (P7, TP7) there was no significant difference in potentials across comparison size. 


\section{Computational Modeling Method}

We evaluate model performance when also including single-trial electrophysiological measurements. The neural correlates of the comparison task, as seen with electroencephalography, include a neural distance effect where the amplitude of event related potentials vary based on the distance of the comparison(e.g., Ben-Shalom, Berger, \& Henik, 2013; Heine, Tamm, Wissmann, \& Jacobs, 2011; Jiang et al., 2010). Our approach for the model predicts the specific responses for each individual participant on each individual trial. This allows the model to account for individual differences in performance.

We focus on the non-symbolic numerical comparison task, which has well cataloged behavioral and neural correlates. Comparisons in which the two numbers are relatively close in value tend to have more errors and longer reaction times. For example, a comparison between 20 and 22 objects produces more frequent errors than a comparison between 20 and 50. This phenomenon is termed the distance effect and depends on the ratio difference between the two values being compared.

The purpose of the computational model is to provide a quantitative description of the governing dynamics of the relevant behavior. We model the hypothesized processes involved in completing numerical tasks. We use this approach so that the model can be used on a wide range of tasks with minimal adjustment. We take an approach that considers the individual differences in participants' behavior in that each is modeled independently. Thus the framework here is that though the basic mechanisms may be the same across individuals, the specifications governing the mechanism may vary. Modeling the sub-processes involved in behavior allows the model to predict behavior on a range of tasks 
that involve similar processes. The long-term goal is to predict individuals' behavior on a range of numerical and arithmetic tasks.

The computational model is instantiated as a multilayered dynamic field theory model (e.g.r Erlhagen \& Schöner, 2002; Sandamirskaya, 2014) that employs neural tuning curves related to numerical cognition (e.g., Prather, 2012, 2014) along with an evolutionary optimization algorithm (see Figure 1). Neural tuning curves correspond to the pattern of activation in a neural population that is associated with a particular stimulus. For example, numerical stimuli have been found to mediate activity in parietal areas in humans and other species (e.g., Nieder \& Miller, 2005; Piazza, Izard, Pinel, Le Bihan, \& Dehaene, 2004). Activity for a given neuron may be selective for a specific number, whereas maximum firing rate is associated with a specific numerical value $\mathrm{N}$, and less for $\mathrm{N}+1, \mathrm{~N}-1$ and so on. This type of coding creates a Gaussian-like neural tuning function (see Equation 1). Each number magnitude is not coded exactly, but in a manner that is consistent with Weber-Fechner's law (Fechner, 1966 [1860]); that noticeable differences between perceptual stimuli are a function of the proportional difference. As the magnitude of the number increases the neural tuning function width increases proportionally. For example, the width of the tuning function for the magnitude 5 is half that of the magnitude 10 , which is half of 20. Thus differences in the perceived value are a function of the proportional stimulus differences, as with Weber-Fechner's law.

The evolutionary algorithm adjusts specifications of the model dynamics to fit each participant's training data. Thus, each participant's data was modeled by a separate and independent instantiation of the computational model. The resulting model specifications were then used to predict the remainder of each participant's data without the further use of the evolutionary algorithm. Initial model input is simply the same stimuli that the participant viewed (exceptions noted later). Model fitness is calculated by comparing model behavior to participants' behavior (see Equation 2). The optimization process 
minimizes the difference between the model and participant behavior (see Equation 3). Model results focus on two questions. First how well does the model fit participant data using the evolutionary algorithm? Second, does using ERP data improve model fit? Analysis of model performance was measured by the deviation between human data and model data. We calculated the proportion of trials in which the model response matched the participant response. The critical comparison is between the proportion of matches. That is if the specifications of the model learned from the training can be used to predict future performance with no reduction in accuracy.

Model Specifications and Procedure. All model instantiations were implemented using MATLAB (Mathworks). The model architecture was a three-level feel forward neural network with the following layers: two input layers, two percept layers, and one decision layer (see Figure 1). Layers modeled neural tuning curves associated with the neural coding of stimuli (e.g., Prather, 2012, 2014; Tudusciuc \& Nieder, 2007). On each trial, the external inputs for the model were the two numerical values to be compared. The two values were represented by proportionally scaled Gaussian curves that reproduce the ratio dependent distance effect. Perceptual layers of the model reproduced the stimuli while activity was forwarded to the decision layer. The internal decision layer connections were specified to produce competition within the layer through lateral inhibition and self-excitation. Thus the two perceptual layers output created competition within the decision layer. This dynamic corresponded to the "decision" which was the index of the first stable activation peak in the decision layer. Each trial was comprised of 500 time steps. The decision layer produced a reliable decision through a steady peak (activity with a peak value at the same layer index for 10 consecutive timesteps). If the model did not resolve within the allotted timesteps, no decision would be made. This is primarily an issue with if there is a large amount of noise in the decision layer. We adjusted the noise to be low enough to avoid a lack of convergence. 
Use of electrophysiological data. We used ERP data as reported in experiment 1 as model input. Data were included the P3, CP3, P7 and TP7 channels as data indicated a significant correlation between ERP amplitude and trial ration difference (i.e., the neural distance effect). For each trial, we calculated the mean ERP amplitude across the time window used in the behavioral experiment. Trial amplitudes were used to predict incorrect responses, which were mostly for trials with ratio differences in the lower half of the range. Thus ERP amplitude only affected model parameters on trials with a ratio difference of 1.7 or less (44 of 90 trials). Since the model relies on trial ERP on only a small subset of trials we included the total participant group $(\mathrm{n}=35)$.

Fitness Calculation and Evolutionary Algorithm Procedure. During training model fit was optimized using an evolutionary algorithm (Equation 3). After determining the specifications of the model that best fit we then used that model for predicting the entirety of participants data without further adjustments via the learning algorithmic. This process is done independently for each participant.

Specifications that varied for each model instantiation included parameters for noise, tuning curve width, the rate of activation change, lateral inhibition. The first generation of the algorithm included 10 instantiations of the model with randomly selected values of the specifications (within a predetermined range). The 10 models are then ranked by the resulting fitness. The bottom 5 performing models were discarded. The best performing model was duplicated for the next generation. Models ranked $2-5$ were mutated, such that their specifications were altered slightly by a random process. The next generation also included 5 new models with randomly selected specifications. This process was repeated over 25 generations to produce a model instantiation that fit the participants' data. Since the model has a small amount of random noise performance does not replicate exactly. To best determine the fitness level of a given set of model specifications we then ran the model repeatedly and calculated the mean fitness level. 
Model fitness is calculated using an overall error term that combines the number of correctly predicted decisions. The overall fit of the model was weighted towards minimizing response prediction error by minimizing both the proportion of total trials correctly predicted (ranging from 0 to 1 ) plus the proportion of incorrect answers which were correctly predicted. We calculate both overall error and separate error for incorrect answers (see Discussion). Since there are far more correct responses made by participants we weight matching an incorrect response more. This helps to avoid the local minimum of the model simply predicting $100 \%$ correct responses.

Model prediction was required to match participant response for each trial specifically, not just the overall performance. For example, the model did not simply predict that a participant might correctly respond to $70 \%$ of trials, the model specifying the response for each trial and must match on each. Thus it is possible for the model to predict correct responses to $70 \%$ of trials but still have some error it the model does not correctly predict which $70 \%$ of trials. This is a much more strict evaluation of the model; it is the difference between predicting "7 of 10 coin flips will be heads" and "the exact sequence HHTHHHTTHH will occur."

\section{Equations}

$$
f(x)=h e^{\frac{-(x-m)^{2}}{2 s^{2}}}
$$

Equation 1. Activation values vectors corresponding to neural tuning curves were calculated using a modified Gaussian distribution function that varies in height similar to a Poisson distribution. The maximum value of the tuning curve $h$, varies with the numerical value $(y)$, such that $h=(121-y)$. The relative width of the calculated curves varied with the value of $S$ (in all current simulations $S=0.7$ ). The mean of the distribution, $m$ is a constant set to 0 . The distance between the target numerical value (T) 
and the current vector index $(\mathrm{V})$ is defined as $X=\log _{20} T-\log _{20} \mathrm{~V}$. The method of defining $X$ by logarithmic differences results in Gaussian functions that are symmetric on a log scale and of identical width.

$$
F=\text { Error }_{\text {Global }}+\text { Error }_{\text {Incorrect }} * Z
$$

Equation 2: Model Fitness Calculation. The error is the proportion of trials that the model correctly predicts. Global Error is for all trials of the current instantiation. Incorrect Error is only for trials in which the participant answered incorrectly. This is the proportion of trials in which the model correctly predicts an incorrect response. This error is weighted by a constant (Z), which is specified in each experiment.

$$
\begin{gathered}
\operatorname{Rank}_{1-2}=I \\
\operatorname{Rank}_{3-5}=\text { Spec }^{*} \text { Rand }_{0.9-1.1}
\end{gathered}
$$

Equation 3: Evolutionary Algorithm. Within each model generation, the 10 model instantiation were ranked by overall fitness. The top two instantiations were carried over to the next generation unchanged. Instantiations ranked $3^{\text {rd }} 4^{\text {th }}$ and $5^{\text {th }}$ were mutated by adjusting each specification by a randomized amount ranging from a $10 \%$ reduction in value to a $10 \%$ increase. Instantiations ranked $6^{\text {th }}$ through $10^{\text {th }}$ were discarded and replaced by novel instantiations in the next generation.

\section{Computational Model Results}


Model fit with no ERP adjustments. Overall error level ranged from 1.73 to 1.24 with a median of 1.60 . Deviations between the model response and participant response, as measured by the proportion of mismatches ranged from 0.23 to 0.08 with a median of 0.11 . Deviations for incorrect trials ranged from 0.75 to 1 with a median of 1 .

Model fit with TP7 channel ERP input. Overall error level ranged from 1.64 to 0.94 with a median of 1.47. Deviations between the model response and participant response, as measured by the proportion of mismatches ranged from 0.78 to 0.05 with a median of 0.14 . Deviations for incorrect trials ranged from 0.25 to 1 with a median of 0.88 .

Model fit with P3 channel ERP input. Overall error level ranged from 1.73 to 1.33 with a median of 1.61 . Deviations between the model response and participant response, as measured by the proportion of mismatches ranged from 0.23 to 0.08 with a median of 0.13 . Deviations for incorrect trials ranged from 0.83 to 1 with a median of 1 .

Model fit with CP3 channel ERP input. Overall error level ranged from 1.73 to 1.24 with a median of 1.6. Deviations between the model response and participant response, as measured by the proportion of mismatches ranged from 0.23 to 0.08 with a median of 0.11 . Deviations for incorrect trials ranged from 0.75 to 1 with a median of 1 . 
Model fit with P7 channel ERP input. Overall error level ranged from 1.68 to 1.12 with a median of 1.48 . Deviations between the model response and participant response, as measured by the proportion of mismatches ranged from 0.50 to 0.05 with a median of 0.12 . Deviations for incorrect trials ranged from 0.44 to 1 with a median of 0.90 .

Comparing the model fit across model instantiations. We compare overall model fit across all five model instantiations. We ran a repeated measure ANOVA across the model instantiation input to evaluate the overall fitness level. We found a significant effect of model input, $F(4,136)=17.29, \mathrm{p}<0.001$. Planned contrast shows that the TP7-input model instantiation had significantly lower error than the no-ERP model instantiation, $\mathrm{t}(34)=5.05, \mathrm{p}<0.0001$. We also found that the P7 channel input model instantiation had a significantly lower error than the no-ERP model instantiation, $t(34)=4.43, p<0.0001$. Neither the P3 channel input model instantiation nor the CP3 channel input model instantiation had significantly lower error than the no-ERP model instantiation, $\mathrm{t}(34)=1.72, \mathrm{p}=0.09$ and $\mathrm{t}(34)=0.35, \mathrm{p}=0.93$ respectively.

\section{General Discussion}

This study demonstrates that a multilayer dynamic field computational model using behavioral and neural data input can accurately predict behavior for individual participants. Specifically, we find that model input from two channels in the parietal area that is associated with numerical processing 
improved model fit beyond what was found with behavioral data. The current model, using independent instantiations and an optimization algorithm was able to fit into a range of participant behavioral data.

In this study, we present a novel computational method for predicting human behavior on a numerical comparison task. The computational method uses both behavioral and neural data to make predictions of participants' future behavior on individual trials. The method may potentially be expanded to include other numerical, arithmetic or general cognitive tasks. We use the canonical non-symbolic numerical task because it is central to the study of numerical cognition and has shown to correlate with other tasks, which are more relevant to educational applications. The question remains if such a modeling approach could be extended to non-symbolic arithmetic, symbolic comparison or even symbolic arithmetic. A similar style neural tuning curve computational model has been used for other tasks such as symbolic number line and symbolic arithmetic (Prather 2012; 2014; 2016). The modeling approach may also be adjusted to predict a particular stimulus set that leads to improved performance on the current task or other general cognitive tasks. Such expanded and adapted use of the computational method could prove to be a significant step in bridging research on cognitive intervention design and individualized training regimens.

Limitations and Future Directions. The overall goal of this modeling approach is to model the subprocesses involved in completing numerical tasks quantitatively. Other modeling approaches, such as drift-diffusion models have been successful in modeling decision-making tasks (Pleskac \& Busemeyer, 2010). Our approach differs by focusing on the potential for broader generalization by modeling the hypothesized processes involved in cognition. The two approaches are not mutually exclusive or in competition. We do not claim that drift-diffusion could not be used to make similar predictions, as there 
are likely multiple computational modeling approaches that could be used towards the similar goals, such as Bayesian models, neural networks, etc.

Evaluating the computational model results requires caution. There are several questions to consider. There was variation in the model fit to training data. Why might some participants have data that is better fit by the computational model? It may be the case that participants with low variability data are better fit by the model. For example, a participant that correctly responded to $100 \%$ of trials would be trivial to predict. Participants performance on the task might correlate with the model fit, where better performing participants had better-fit models. A correlation between participant performance and model fit was significant $\mathrm{R}=-0.54, \mathrm{t}(22)=3.009, \mathrm{p}<0.01$. We then looked more specifically of model predictions of incorrect answers. Model performance in predicting incorrect responses of participants did not correlate with participant performance, $R=-0.22, t(22)=1.05, p=0.30$. The model's performance somewhat depends on the characteristics of the participant data. The initial model fit was best for participants with a high degree of accuracy. However model prediction of which trials the participants answered incorrectly was not correlated with participant performance.

It is unclear if there is a limitation to the number of trials the model could predict based on the training. There may be an in principal limitation to the number of trials the model can predict. It may also be the case that a more extensive training set for the model results in more accurate extrapolation. Perhaps a multiple visit experiment with a much larger number of trials would result in greater accuracy in predictions. The current study only involved a brief experimental session. Longer and potentially multiple visit experimental sessions could address this question. 



\section{References}

Anguera, J. a, Boccanfuso, J., Rintoul, J. L., Al-Hashimi, O., Faraji, F., Janowich, J., ... Gazzaley, a. (2013). Video game training enhances cognitive control in older adults. Nature, 501(7465), 97-101. http://doi.org/10.1038/nature12486

Ben-Shalom, T., Berger, A., \& Henik, A. (2013). My brain knows numbers! - an ERP study of preschoolers' numerical knowledge. Frontiers in Psychology, 4(October), 716.

http://doi.org/10.3389/fpsyg.2013.00716

Chen, Q., \& Li, J. (2014). Association between individual differences in non-symbolic number acuity and math performance: a meta-analysis. Acta Psychologica, 148, 163-72. http://doi.org/10.1016/j.actpsy.2014.01.016

Cohen Kadosh, R., Dowker, A., Heine, A., Kaufmann, L., \& Kucian, K. (2013). Interventions for improving numerical abilities: Present and future. Trends in Neuroscience and Education, 2(2), 85-93. http://doi.org/10.1016/j.tine.2013.04.001

Erlhagen, W., \& Schöner, G. (2002). Dynamic field theory of movement preparation. Psychological Review, 109(3), 545-572. http://doi.org/10.1037//0033-295X.109.3.545

Fazio, L. K., Bailey, D. H., Thompson, C. a, \& Siegler, R. S. (2014). Relations of different types of numerical magnitude representations to each other and to mathematics achievement. Journal of Experimental Child Psychology, 123, 53-72. http://doi.org/10.1016/j.jecp.2014.01.013

Ginsburg, H. (2003). Test of Early Mathematics Ability - 3rd Edition. Austin, TX: Pro-Ed.

Heine, A., Tamm, S., Wissmann, J., \& Jacobs, A. M. (2011). Electrophysiological correlates of non-symbolic numerical magnitude processing in children: joining the dots. Neuropsychologia, 49(12), 3238-46. 
http://doi.org/10.1016/j.neuropsychologia.2011.07.028

Iuculano, T., Rosenberg-Lee, M., Richardson, J., Tenison, C., Fuchs, L., Supekar, K., \& Menon, V. (2015). Cognitive tutoring induces widespread neuroplasticity and remediates brain function in children with mathematical learning disabilities. Nature Communications, 6, 8453. http://doi.org/10.1038/ncomms9453

Jiang, T., Qiao, S., Li, J., Cao, Z., Gao, X., Song, Y., ... Chen, C. (2010). Effects of symbol type and numerical distance on the human event-related potential. Neuropsychologia, 48(1), 201-10. http://doi.org/10.1016/j.neuropsychologia.2009.09.005

Libertus, M. E., Feigenson, L., \& Halberda, J. (2013). Is Approximate Number Precision a Stable Predictor of Math Ability? Learning and Individual Differences, 25, 126-133. http://doi.org/10.1016/j.lindif.2013.02.001

Libertus, M. E., Odic, D., \& Halberda, J. (2012). Intuitive sense of number correlates with math scores on college-entrance examination. Acta Psychologica, 141(3), 373-379. http://doi.org/10.1016/j.actpsy.2012.09.009

Mazzocco, M. M., Feigenson, L., \& Halberda, J. (2011). Impaired Acuity of the Approximate Number System Underlies Mathematical Learning Disability ( Dyscalculia ), 82(4), 1224-1237. http://doi.org/10.1111/j.1467-8624.2011.01608.x

Nieder, A., \& Miller, E. K. (2005). Neural Correlates of Numerical Cognition in the Neocortex of nonhuman primates. In Cognition.

Park, J., Bermudez, V., Roberts, R. C., \& Brannon, E. M. (2016). Journal of Experimental Child Non-symbolic approximate arithmetic training improves math performance in preschoolers. Journal of 
Experimental Child Psychology, 152, 278-293. http://doi.org/10.1016/j.jecp.2016.07.011

Park, J., \& Brannon, E. M. (2013). Training the approximate number system improves math proficiency. Psychological Science, 24(10), 2013-9. http://doi.org/10.1177/0956797613482944

Park, J., \& Brannon, E. M. (2014). Improving arithmetic performance with number sense training: An investigation of underlying mechanism. Cognition, 133(1), 188-200. http://doi.org/10.1016/j.cognition.2014.06.011

Piazza, M., Izard, V., Pinel, P., Le Bihan, D., \& Dehaene, S. (2004). Tuning curves for approximate numerosity in the human intraparietal sulcus. Neuron, 44(3), 547-55. http://doi.org/10.1016/j.neuron.2004.10.014

Pleskac, T. J., \& Busemeyer, J. R. (2010). Two-stage dynamic signal detection: a theory of choice, decision time, and confidence. Psychological Review, 117(3), 864-901. http://doi.org/10.1037/a0019737

Prather, R. W. (2012). Connecting neural coding to number cognition: a computational account. Developmental Science, 15(4), 589-600. http://doi.org/10.1111/j.1467-7687.2012.01156.x

Prather, R. W. (2014). Numerical discrimination is mediated by neural coding variation. Cognition, 133(3), 601-610. http://doi.org/10.1016/j.cognition.2014.08.003

Prather, R. W., \& Alibali, M. W. (2011). Children's Acquisition of Arithmetic Principles: The Role of Experience. Journal of Cognition and Development, 12(3), 332-354. http://doi.org/10.1080/15248372.2010.542214

Prather, R. W., \& Heverly-Fitt, S. (2016). Neural coding partially accounts for the relationship between children's number-line estimation and number comparison performance. Frontiers in Developmental Psychology. 
Sandamirskaya, Y. (2014). Dynamic neural fields as a step toward cognitive neuromorphic architectures. Frontiers in Neuroscience, 7(January), 1-13. http://doi.org/10.3389/fnins.2013.00276

Tudusciuc, 0., \& Nieder, A. (2007). Neuronal population coding of continuous and discrete quantity in the primate posterior parietal cortex. Neuron, 104(36).

Vanlehn, K. (2011). The Relative Effectiveness of Human Tutoring , Intelligent Tutoring Systems , and Other Tutoring Systems The Relative Effectiveness of Human Tutoring , Intelligent Tutoring Systems , and Other Tutoring Systems, (August 2014), 37-41.

http://doi.org/10.1080/00461520.2011.611369 


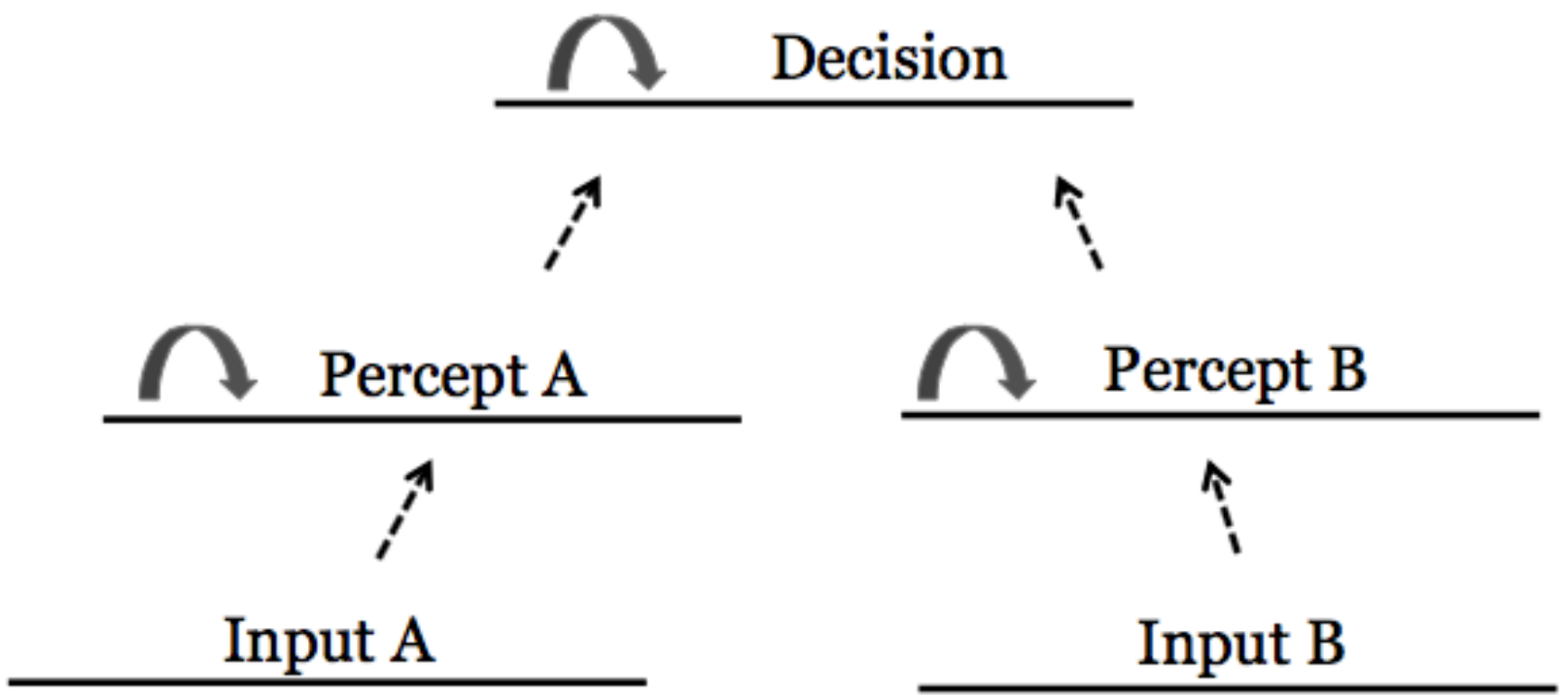

Figure 1. Schematic of computational model architecture. Pairs (A and B) are represented by activation patterns in layers Input A and Input B. Activity in those layers projects to Percept A and Percept B layers. Percept layers include inhibitory self connections and excitatory connections to the Decision layer. 



\section{Appendix A}

Stimuli List

$25 \quad 35$

$50 \quad 75$

$25 \quad 30$

$40 \quad 42$

$25 \quad 65$

$35 \quad 91$

$25 \quad 35$

$23 \quad 23$

$14 \quad 26$

$19 \quad 42$

$20 \quad 22$

$13 \quad 29$

$30 \quad 30$

$10 \quad 18$

$15 \quad 21$

$50 \quad 75$

$15 \quad 35$

$10 \quad 19$

$15 \quad 35$
$29 \quad 70$

$30 \quad 33$

$19 \quad 44$

$20 \quad 32$

$10 \quad 20$

$10 \quad 18$

$15 \quad 33$

$30 \quad 51$

$15 \quad 21$

$14 \quad 14$

$24 \quad 60$

$30 \quad 45$

$10 \quad 19$

$23 \quad 50$

$29 \quad 70$

$24 \quad 60$

$20 \quad 30$

$30 \quad 39$

$30 \quad 33$

$10 \quad 15$

$35 \quad 56$

$22 \quad 54$
$14 \quad 28$

$20 \quad 52$

$30 \quad 30$

$15 \quad 33$

$10 \quad 25$

$20 \quad 52$

$22 \quad 54$

$20 \quad 38$

$36 \quad 87$

$20 \quad 32$

$30 \quad 51$

$14 \quad 19$

$15 \quad 18$

$30 \quad 39$

$20 \quad 34$

$20 \quad 30$ 


$\begin{array}{llllll}10 & 20 & 25 & 30 & 19 & 44 \\ 40 & 42 & 40 & 60 & 10 & 13 \\ 35 & 56 & 10 & 11 & 25 & 65 \\ 10 & 13 & 23 & 50 & 40 & 60 \\ 20 & 38 & 10 & 15 & 20 & 22 \\ 16 & 40 & 36 & 87 & 23 & 23 \\ 20 & 34 & 19 & 42 & 13 & 29 \\ 10 & 25 & 45 & 54 & 45 & 54 \\ 14 & 19 & 14 & 28 & 14 & 26 \\ 30 & 45 & 15 & 18 & 14 & 14 \\ 35 & 91 & 16 & 40 & 10 & 11\end{array}$

